\title{
Criminologie
}

\section{Les réfugiés à notre porte : violence organisée et souffrance sociale}

\section{Cécile Rousseau}

Volume 33, numéro 1, printemps 2000

La victimologie : quelques enjeux

URI : https://id.erudit.org/iderudit/004743ar

DOI : https://doi.org/10.7202/004743ar

Aller au sommaire du numéro

\section{Éditeur(s)}

Les Presses de l'Université de Montréal

ISSN

0316-0041 (imprimé)

1492-1367 (numérique)

Découvrir la revue

\section{Citer cet article}

Rousseau, C. (2000). Les réfugiés à notre porte : violence organisée et souffrance sociale. Criminologie, 33(1), 185-201.

https://doi.org/10.7202/004743ar

\section{Résumé de l'article}

Beaucoup de réfugiés qui s'établissent dans les pays occidentaux ont vécu des expériences traumatiques en lien avec la situation de violence organisée qui prévaut dans leur pays d'origine. La souffrance qui résulte de ces expériences est souvent interprétée dans le pays d'asile à partir d'un modèle médical qui se centre sur les manifestations psychopathologiques chez les individus et en particulier sur la présence du syndrome de stress post-traumatique. Une telle approche peut avoir pour effet de normaliser la pathologie sociale en en faisant porter le poids à l'individu et de pathologiser les réponses psychologiques et physiologiques normales à la terreur.Cet article propose de repenser le traumatisme en tant que processus de métamorphose qui génère à la fois des forces et des difficultés sur le plan personnel et collectif. Au travers de dynamiques très diverses la violence organisée vise la rupture du lien social. En provoquant un sentiment d'absurdité elle désorganise le sentiment de cohérence des personnes, des familles et des communautés. La dissociation entre les aspects individuels et collectifs de la violence organisée qui prévaut chez les professionnels et institutions du pays hôte peut aggraver la fragmentation des liens sociaux. Un processus de reconstruction se bâtit dans le va-et-vient entre l'élaboration d'un réseau de significations et le rétablissement du lien social. Le travail de construction du sens se fait d'abord et avant tout dans les familles et au sein du groupe auquel le réfugié s'identifie. Il importe alors surtout de savoir ne pas intervenir afin de ne pas interférer avec les stratégies mises de l'avant par la communauté. Parfois cependant, devant les limites ou l'échec des stratégies disponibles, une intervention extérieure impliquant les instances de la société hôte se justifie. Dans un contexte d'exil les équipes cliniques peuvent jouer un rôle de médiation important en permettant d'une part aux familles réfugiées de se réapproprier et de valider les stratégies traditionnelles ou politiques qui leur sont disponibles dans le pays d'origine et, d'autre part, en facilitant la co-construction de solutions métisses qui reflètent la multiplicité des univers dans lesquels naviguent les réfugiés.
Ce document est protégé par la loi sur le droit d'auteur. L'utilisation des services d’Érudit (y compris la reproduction) est assujettie à sa politique d'utilisation que vous pouvez consulter en ligne.

https://apropos.erudit.org/fr/usagers/politique-dutilisation/ 


\title{
Les réfugiés à notre porte : violence organisée et souffrance sociale
}

\author{
Cécile Rousseau \\ Professeure \\ Directrice \\ Département de psychiatrie \\ Université McGil • Canada, \\ Équipe de psychiatrie transculturelle, Hôpital de Montréal pour enfants \\ micr@musica.mcgill.ca
}

RÉsumé - Beaucoup de réfugiés qui s'établissent dans les pays occidentaux ont vécu des expériences traumatiques en lien avec la situation de violence organisée qui prévaut dans leur pays d'origine. La souffrance qui résulte de ces expériences est souvent interprétée dans le pays d'asile à partir d'un modèle médical qui se centre sur les manifestations psychopathologiques chez les individus et en particulier sur la présence du syndrome de stress post-traumatique. Une telle approche peut avoir pour effet de normaliser la pathologie sociale en en faisant porter le poids à l'individu et de pathologiser les réponses psychologiques et physiologiques normales à la terreur.

Cet article propose de repenser le traumatisme en tant que processus de métamorphose qui génère à la fois des forces et des difficultés sur le plan personnel et collectif. $\mathrm{Au}$ travers de dynamiques très diverses la violence organisée vise la rupture du lien social. En provoquant un sentiment d'absurdité elle désorganise le sentiment de cohérence des personnes, des familles et des communautés. La dissociation entre les aspects individuels et collectifs de la violence organisée qui prévaut chez les professionnels et institutions du pays hôte peut aggraver la fragmentation des liens sociaux. Un processus de reconstruction se bâtit dans le va-et-vient entre l'élaboration d'un réseau de significations et le rétablissement du lien social. Le travail de construction du sens se fait d'abord et avant tout dans les familles et au sein du groupe auquel le réfugié s'identifie. Il importe alors surtout de savoir ne pas intervenir afin de ne pas interférer avec les stratégies mises de l'avant par la communauté. Parfois cependant, devant les limites ou l'échec des stratégies disponibles, une intervention extérieure impliquant les instances de la société hôte se justifie. Dans un contexte d'exil les équipes cliniques peuvent jouer un rôle de médiation important en permettant d'une 
part aux familles réfugiées de se réapproprier et de valider les stratégies traditionnelles ou politiques qui leur sont disponibles dans le pays d'origine et, d'autre part, en facilitant la co-construction de solutions métisses qui reflètent la multiplicité des univers dans lesquels naviguent les réfugiés.

RÉSUMÉ - Many refugees who become residents of western countries have lived through traumatic experiences linked to the situation of organized violence that prevails in their countries of origin. The suffering that results from these experiences is often interpreted, in the country of asylum, based on a medical model which focuses on the psychopathological symptoms of the individual and particularly on Post Traumatic Stress Disorder (PTSD). Such an approach may have the effect of normalising social pathology by making the individual carry the weight of it and by pathologizing a normal psychological and physiological response to terror.

This article proposes to rethink trauma, seeing it as a process of metamorphosis which generates both strengths and difficulties on an individual and a collective basis. Disassociating the individual and collective aspects of organized violence, a practice which prevails among professions and institutions of the host countries, may aggravate the fragmentation of social connections. A reconstruction process takes place in the coming and going between the elaboration of a network of significances and the reestablishment of social connections. The work of construction of sense takes place first and foremost within families and in the group with which the refugee identifies. It matters then especially not to intervene so as not to interfere with the strategies employed by the community. At times, however, faced with limitations or the failure of available strategies, external intervention by the host society is justified. In a context of exile, clinical teams may play an important mediation role by allowing refugee families to reappropriate and validate their traditional strategies or those political strategies that are available to them in their countries of origin, and by facilitating the joint construction of solutions that reflect the multiple universes in which the refugees navigate.

RESUMEN - Numerosos refugiados establecidos en países occidentales han vivido experiencias traumáticas a causa de la violencia organizada que prevalece en el país de origen. El sufrimiento que resulta de estas experiencias suele interpretarse en los paises de asilo a partir de un modelo médico centrado en las diversas manifestaciones psicopatológicas encontradas en estos indivíduos, en particular la presencia del síndrome de stress post-traumático. La aplicación de este enfoque puede tener por efecto la normalización de la patología social al descargar el peso de la misma sobre el indivíduo, a la vez que convierte en patológicas las respuestas de orden psicológico y fisiológico completamente normales ante el terror.

El presente artículo propone replantear la experiencia traumática en tanto que proceso de metamorfosis capaz de producir simultáneamente fortalezas y dificultades, tanto en el plano personal como colectivo. Mediante diversas dinámicas, la violencia organizada se propone lograr la ruptura del vínculo social. De esta manera, al provocar un sentimiento de absurdidad se desorganiza el sentimiento de coherencia tanto en las personas, como en las familias y en las propias comunidades. La separación entre los aspectos individuales y colectivos que integran la violencia organizada y que prevalece 
entre los profesionales y las instituciones del país receptor, puede àgravar la fragmentación de los lazos sociales. Un proceso de reconstucciòn se basa en el intercambio entre la elaboración de significaciones y el restablecimiento del nexo social. El trabajo de construcción del sentido es llevado a cabo primero y sobre todo dentro de las familias y en el seno del grupo con el cual se identifica el refugiado. Es importante, en consecuencia, saber no intervenir si se quiere evitar la interferencia en las estrategias iniciadas por la comunidad. No obstante, algunas veces ante las limitaciones o el fracaso de las estrategias disponibles, pareciera justificarse una intervención exterior por parte de las instancias de la sociedad receptora. En el contexto del exilio, los equipos clínicos pueden desempeñar un papel de mediación importante, permitiendo por una parte a las familias refugiadas apropiarse de nuevo y validar las estrategias tradicionales o políticas disponibles en su país de origen, y por otra, facilitando la co-construcción de soluciones mestizas que reflejen la multiplicidad de universos en los cuales suelen navegar los refugiados.

\section{Introduction}

L'Occident a fait de la violence et de ses conséquences un problème porté par des individus, qu'ils soient des agresseurs qu'il faut comprendre, soigner, contrôler et punir ou des victimes qui, par ce statut, accèdent à des droits et à la réparation du tort qui leur a été fait. La transmission traumatique, autour de l'idée que la violence engendre la violence, établit un trait d'union entre les deux groupes. Dans cette perspective, la recherche de sens et l'élaboration d'étiologies pouvant fonder des programmes de prévention se sont de plus en plus tournées vers des déterminants individuels, qu'ils soient génétiques ou qu'ils relèvent d'une histoire d'abus et de problèmes familiaux.

La tache aveugle de ce discours est la violence organisée, c'est-à-dire la violence exercée par un groupe d'humains contre un autre groupe à cause de ses caractéristiques politiques, raciales, religieuses, ethniques, sociales, sexuelles ou autres. Pour nommer cette violence collective extrême, on parle souvent de profonde inhumanité, comme si elle ne caractérisait pas justement, même partiellement, notre condition humaine. Cette négation parle sans doute de notre besoin de distance, de notre désir de renvoyer dans l'innommable, ou en tout cas dans le « nonmoi » la guerre, la torture et la capacité infinie des hommes à faire souffrir d'autres hommes. La distance entre nous et la violence organisée se renforce dans une métaphore géographique où le « là-bas », le monde non occidental, devient porteur de toute la barbarie du monde, des dictatures aux intégrismes. Or, comme le rappelle si bien De Certeau 
(1987) en parlant de la torture, celle-ci et les autres formes de violence collective ne sont pas un restant d'époques révolues mais sont, au contraire, le produit de la violence " propre " de notre modernité. Certes, dans nos sociétés, cette violence propre emprunte des formes politiquement correctes moins directement reconnaissables, mais qui ne sont pas nécessairement moins pernicieuses que les violences mises en place pour des systèmes ouvertement répressifs qui légitiment la résistance « contre ».

Kleinman et Kleinman (1997) proposent de penser les conséquences de la violence organisée en termes de souffrance sociale. Celle-ci met en évidence, d'abord et avant tout, le lien étroit entre : les problèmes personnels et sociaux ; l'individuel et le collectif ; la psychopathologie, l'idéologie et la politique. Or, pour ces auteurs, les processus culturels d'analyse technique et rationnelle qui décrivent la souffrance sociale et les interventions bureaucratiques qui leur correspondent ne font trop souvent qu'aggraver cette souffrance. Une méconnaissance des enjeux complexes pour chacun des groupes affectés est souvent à la source de ces erreurs. Trop fréquemment, l'intervention sert à normaliser la pathologie sociale en en faisant porter le poids à l'individu, quand elle ne pathologise pas les réponses psychologiques et physiologiques normales à la terreur.

Les réfugiés qui arrivent dans les pays occidentaux fuient la violence organisée de leur pays d'origine, que ce soit la guerre, la persécution ou les conditions sociales et économiques générées par celles-ci. Une fois dans le pays hôte, ils se retrouvent coincés entre un discours idéal sur l'asile, la réalité d'une opinion publique souvent hostile et des politiques migratoires contraignantes qui les forcent à prouver qu'ils ne sont pas des fraudeurs ou des criminels (Moreau et al., 1999). Les réfugiés vivent donc un double traumatisme : le premier, dans leur pays d'origine, provient le plus souvent de la volonté de destruction de ce qu'ils sont ou de ce qu'ils représentent comme groupe ; le deuxième vient du déni de ce qu'ils ont vécu par le pays hôte (Rousseau et al., 1992), qui se traduit soit par une banalisation de leur expérience, soit par un discours sur les faux réfugiés et le mensonge. Les réfugiés deviennent alors les seuls auteurs de l'histoire d'horreur qu'ils portent : l'absurdité et le non-sens règnent.

Dans ce contexte, l'intervention qui va s'organiser autour d'eux dans le pays hôte peut, par un recours à des catégories clivées, figer les réfugiés dans une position de victimes passives ou, dans certains cas, d'agresseurs potentiels. Mais l'intervention peut aussi, à partir d'un 
rétablissement de liens sociaux même ténus et en se fondant sur les forces des personnes, réhabiliter une position de sujet qui permet de sortir de la paralysie traumatique, de retrouver le mouvement de la vie.

Dans les dernières décennies environ 15000 réfugiés sont arrivés chaque année au Canada, s'établissant principalement à Toronto, Montréal et Vancouver. Leur provenance varie avec le temps : aux vagues d'exodes de l'Amérique latine et de l'Asie du Sud-Est ont succédé des populations provenant plus de l'Asie du Sud (Bangladesh, Sri Lanka, Pakistan), d'Europe de l'Est et de Russie. Enfin, les derniers conflits africains ont amené d'importants contingents de Somaliens, d'Algériens et de personnes provenant de la région des Grands Lacs africains et cherchant asile au Canada.

Dans le cadre de cet article, nous utiliserons les concepts de violence organisée et de souffrance sociale pour décrire, dans une perspective clinique, le lien entre l'expérience personnelle et collective des réfugiés dans les pays occidentaux, en nous référant de façon plus spécifique à la situation canadienne. Après avoir rappelé rapidement certaines des prémisses occidentales au sujet de l'impact de la violence et des traumatismes pour les individus, nous aborderons la nécessité d'élargir ce cadre de pensée pour saisir aussi les conséquences de la violence organisée pour les réfugiés au plan collectif. Nous aborderons ensuite la question de la reconstruction du sens dans le pays hôte et le rôle éventuel que peuvent y avoir les intervenants du pays hôte par des programmes d'intervention et de prévention.

\section{Les prémisses occidentales : la construction du sens centrée sur l'individu}

Une première prémisse occidentale concerne la construction de la notion de victime. Un rapide survol de la littérature scientifique et des divers projets d'intervention dans des situations de violence massive ne laisse subsister aucun doute (Summerfield, 1998) : nous voulons tous aider les victimes, la souffrance des agresseurs étant à la limite, quoique de façon implicite, considérée comme un juste châtiment. Mais comment, lorsque des communautés entières s'affrontent, départage-ton les victimes des agresseurs? À partir des faits? Du discours? Des idées, voire des intentions? Quels que soient les intérêts précis qui ont présidé au déclenchement d'un conflit, l'internalisation de la violence par une société agrandit cette zone grise où tout humain est à la fois victime et 
agresseur, menacé et menaçant. Construire de façon extérieure, et nécessairement idéologique, des programmes de protection qui s'adressent aux victimes de la violence organisée n'est donc pas un geste neutre, puisque ces programmes peuvent renforcer non seulement les clivages qui existent sur la scène internationale et nationale, mais aussi ceux qui existent en chaque acteur, fût-il passif, du conflit (Marotte, 1999).

Paradoxalement, une deuxième prémisse construit la notion de traumatisme dans le champ médical en effaçant la distinction, pourtant très importante dans le discours social, entre victimes et agresseurs. Ce paradoxe a une origine historique : après la guerre au Viêt-nam, la nécessité de réintégrer socialement des vétérans que l'on ne pouvait considérer ni comme des héros ni comme de pures victimes forçait l'adoption d'une catégorie diagnostique qui permettait de définir le traumatisme comme une maladie, tout en effaçant les autres niveaux de sens qui y étaient rattachés (Young, 1995). L'idée que le traumatisme produit avant tout un handicap sous forme d'une psychopathologie individuelle, décrite dans le syndrome de stress post-traumatique (SSPT), domine les travaux nordaméricains sur le sujet. Une métaanalyse des travaux portant sur des traumatismes divers montre pourtant que le SSPT n'est présent que chez $20 \%$ des personnes ayant subi l'un ou l'autre de ces traumatismes (Yehuda et McFarlane, 1995). Qu'est-ce à dire pour les autres $80 \%$ ? Ces personnes ne présentent-elles aucune conséquence de leur traumatisme? Souffrentelles d'autres conséquences psychopathologiques (Pynoos et Nader, 1993) ou, comme le suggèrent Macksoud et al. (1993), le traumatisme peut-il aussi être associé à l'apparition de forces comme l'altruisme ou la capacité de planifier, sans que ce phénomène ne soit documenté dans des études centrées essentiellement sur la psychopathologie?

S'opposant à la conception dominante qui construit le traumatisme comme un handicap, Nathan (1988) propose de le concevoir comme un processus de métamorphose qui transforme la personne au travers de la souffrance et génère à la fois des forces et des difficultés. Cette conceptualisation s'applique également à la transformation des collectivités par la violence organisée. Le clivage entre les littératures psychologiques et médicales d'une part et de sciences sociales d'autre part donne cependant accès à peu de théorisation au sujet du lien entre les conséquences individuelles et collectives du traumatisme.

La perception de la souffrance des réfugiés au travers de catégories occidentales peut donc générer des difficultés en individualisant le problème et en faisant porter aux réfugiés le poids de la psychopatho- 
logie supposée en découler dans un contexte qui ne reconnaît pas ou peu les ressources et les stratégies personnelles et collectives qui peuvent émerger de la souffrance même extrême.

\section{L'empreinte du traumatisme chez les réfugiés : expérience personnelle et collective}

$\mathrm{Au}$ travers de dynamiques qui peuvent prendre des formes très diverses, la violence organisée est d'abord et avant tout un traumatisme de nonsens, c'est-à-dire qu'elle provoque un sentiment d'absurdité et désorganise la cohérence de l'univers personnel (Marotte, 1995). Même après l'exil, c'est aux niveaux de la personne, de la famille et de la communauté, les trois instances qui composent cet univers, qu'il faut saisir l'empreinte du traumatisme chez le réfugié.

Sur le plan personnel la souffrance provoquée par la violence organisée va osciller entre deux pôles : celui de l'évitement et celui de la répétition (Terr, 1991). Du côté de la répétition on retrouve bien sûr les cauchemars qui sont l'un des marqueurs traumatiques les plus reconnus mais qui ne sont pas universellement considérés comme des signes de psychopathologie. Rechtman (1992) observe, en effet, que pour les Khmers les cauchemars peuvent représenter le retour de personnes malmortes parce qu'elles n'ont pas pu être honorées suivant les rituels bouddhistes après leur décès. Les phénomènes de reviviscence envahissants et les jeux traumatiques chez les enfants se situent aussi parmi les phénomènes de répétition. De même, la suractivation autonomique et l'état d'alerte perpétuel constituent l'inscription corporelle de la répétition, la mémoire du corps.

À la répétition, mémoire incessante et douloureuse qui s'impose comme un signal d'alarme persistant, comme une blessure qui ne peut guérir, s'oppose l'évitement. Celui-ci peut être total, comme dans la dissociation complète mais, le plus souvent, il est partiel et prend des formes diverses : oubli, évitement plus ou moins conscient de stimuli rappelant le trauma, émoussement émotionnel. Au niveau des liens intimes et familiaux, la distance émotionnelle qui résulte de l'ébranlement des liens fondamentaux de confiance en l'humain est le premier maillon représentant le bris du lien social.

Bien sûr tous ces symptômes font partie du SSPT mais ils n'ont pas un sens univoque et doivent être pensés tels que perçus et construits dans un contexte et une culture spécifiques qui donnent un sens à ce qui s'est 
passé, influencent les formes d'expression que va prendre la souffrance et proposent des avenues de reconstruction.

\section{L'éléphant canadien triste : histoire d'Ali}

Ali est un jeune garçon algérien de 8 ans que son école a référé à notre clinique parce qu'il est très retiré. Solitaire et rêveur, il est sans cesse distrait et oublie tout : ses devoirs bien sûr, mais aussi ses habits, même l'biver. Ali est le cinquième d'une famille de sept enfants. Il a toujours été considéré par sa mère comme un enfant très spécial parce qu'à sa naissance il était légèrement blessé à un œil. Plus proche de sa mère qu'aucun autre mais aussi plus vulnérable, Ali est resté avec elle les deux premières années de sa vie. Vers l'âge de trois ans, alors que ses grands-parents le ramenaient chez lui par un sentier poussiéreux à la tombée du jour, Ali est devenu très agité, terrifié et inconsolable. Un iman consulté a diagnostiqué la possession par un djinn et Ali, traité de façon traditionnelle, s'est rétabli. Deux ans plus tard, à l'âge de cinq ans, ses parents, devant fuir la violence en Algérie, laissent derrière eux leurs enfants. Ali a mal vécu cette séparation. "Ali est triste " écrivaient les grands-parents. Durant ces deux années de séparation, Ali a été le plus exposé au traumatisme. À deux reprises, c'est lui qui a trouvé des corps égorgés jetés devant la porte de la maison des grands-parents. Puis Ali a été kidnappé pendant deux jours, de cela il n'a jamais pu parler, jamais... Quand finalement Ali a rejoint ses parents au Canada, c'était un autre enfant, tour à tour terrifié ou absent.

Lors de la première rencontre à la clinique, Ali accepte de rester quelques moments seul avec nous. Son anxiété est palpable. Je lui propose de dessiner pour lui, il acquiesce et se tait ; l'anxiété encore. Je lui demande :

- Je dessine quelque chose de l'Algérie ou du Canada?

Ali très vite:

- Du Canada.

- Comme quoi?

- Un éléphant!

Alors je dessine un éléphant canadien.

- Il se sent comment l'éléphant?

- Il est fâché... non, non il est triste.

Je dessine des larmes.

- Non, il est triste mais il ne pleure pas.

- Pourquoi est-il triste?

- Parce qu'il ne peut pas parler... 
La douleur et le silence d'Ali sont un écho de la douleur de son père dont le père s'est fait égorger dix jours plus tôt, un écho aussi de la douleur et du silence de sa communauté fragmentée.

À la suite de cette première consultation le père va s'occuper de son fils en lui récitant des versets du Coran pour chasser la peur et amener la paix, comme lorsque le djinn avait attaqué Ali. Ali viendra aussi en thérapie pour " pouvoir parler » et il commencera tranquillement à dire et à faire confiance. Maintenant il se permet même de se fâcher... terriblement.

Pour Ali, la vie qui reprend se tricote entre de multiples univers : le monde des djinns, le lien à Dieu, la psychothérapie occidentale, l'école où l'on respecte mieux son silence. Dans sa famille il incarne à la fois la déchirure de son peuple et l'espoir d'un lendemain qui pourrait malgré tout être meilleur.

Au plan collectif, par-delà la destruction du corps physique, l'inversion du monde des valeurs et l'absurdité, la violence organisée vise la rupture du lien social (Vinar et Vinar, 1989). Certains auteurs, comme les thérapeutes des mères de la Place de Mai en Argentine, insistent sur l'imposition d'un silence social par la terreur (Kordon et al., 1992). D'autres abordent surtout l'effritement de tout le tissu social par une situation de violence chronique où la méfiance devient une stratégie de survie incontournable (Mekki-Berrada et al., soumis). Taussig (1992) avance que la violence organisée s'attaque à la mémoire collective, dissolvant les liens sociaux à cause de l'impossibilité d'être en continuité avec une histoire et donc de créer une appartenance collective. Or, la dissociation entre les aspects individuels et collectifs de la violence organisée qui prévaut chez les professionnels et institutions du pays hôte aggrave souvent indirectement la fragmentation des liens sociaux. Le réfugié qui a été considéré comme déviant par le pouvoir de son pays d'origine, se voit confirmé dans ce statut par les intervenants du pays d'accueil qui le déclarent malade et lui assignent un diagnostic sans dénoncer les auteurs de la violence collective qu'il a subie (Becker et al., 1990). Un processus de reconstruction doit se penser dans le va-et-vient entre le personnel et le collectif, l'ici et le là-bas, le passé et le futur.

\section{La construction du sens et le rétablissement du lien social}

Pour pouvoir continuer à vivre, le réfugié a besoin de sortir de l'absurde et de retrouver un univers signifiant, complexe et polysémique. Pour 
certaines communautés, l'élaboration du sens peut se bâtir sur l'histoire traumatique qui constitue le point tournant entre l'avant et l'après. Malgré la douleur qui l'accompagne, la mémoire, personnelle et collective, devient alors une nécessité, pour assurer le « jamais plus » et transformer la blessure du traumatisme en garantie de sécurité pour les générations à venir (Primo-Lévi, 1995 ; Kirmayer, 1996). Dans d'autres collectivités le sens se bâtit autour du traumatisme, en établissant une continuité entre un avant et un après par-delà le traumatisme qui reste essentiellement dans le non-dit (Rousseau et al., sous presse). L'évitement et l'atomisation du temps (Beiser et al., 1998) servent alors de stratégie collective et la trame de significations puise dans un passé de valeurs et de rituels qui ont survécu au traumatisme sans toutefois évoquer directement celui-ci (Skidmore, 1994).

Le partage d'un sens permet la sortie de l'absurde et un premier rétablissement du lien social, qui peut être un lien avec des figures faisant partie de la réalité directe du réfugié ou un lien avec des figures internalisées qu'il s'agisse d'êtres chers, d'ancêtres ou de dieu. On considère trop souvent que les réfugiés sont automatiquement protégés par un contact avec des ressortissants de leur communauté d'origine qui sont perçus comme une source de soutien. Le réfugié et le groupe que la société hôte identifie comme étant sa communauté d'appartenance peuvent cependant comprendre le passé de façon très divergente et la mise en évidence de cette divergence de sens peut parfois désorganiser plus le réfugié que sa solitude :

Pedro est un jeune homme salvadorien qui était professeur dans son pays et organisait du théatre engagé, aussi appelé "de protesta ». Il avait été emprisonné et torturé par les autorités militaires et avait fait un long voyage à pied pour quitter son pays et traverser l'Amérique centrale puis gagner les États-Unis. Il avait traversé avec succès toutes ces difficultés et était arrivé à Montréal. Là il avait rencontré des jeunes de son pays avec lesquels il avait fraternisé. Pedro, se sentant enfin en sécurité, avait proposé d'organiser des activités théâtrales. Au cours de ces activités, le groupe s'était subitement retourné contre lui, le traitant de "guerillero ». Pedro s'était alors effondré et était devenu extrêmement déprimé et suicidaire. Il avait dû être hospitalisé en psychiatrie. Il avait réussi à surmonter des traumas et des difficultés hors du commun, survécu à la torture et à la famine, mais il n'avait pas supporté de sentir que ceux qu'il identifiait comme « les siens » le reniaient. En se désolidarisant de lui, ses pairs avaient fait éclater son monde que les militaires, malgré leurs efforts, n'avaient pu détruire.

L'absurdité s'immisce parfois jusqu'au sein des familles. La situation des couples d'origine ethnique mixte en ex-Yougoslavie ou au Rwanda 
en est un exemple douloureux. Pour résister aux haines bâties collectivement autour de la famille, il est parfois nécessaire d'invoquer des niveaux de signification différents de ceux mis de l'avant par les groupes d'appartenance de ses membres :

Juan et Lucia sont guatémaltèques. Quand ils se sont mariés, Juan était déjà très impliqué auprès d'organisations politiques révolutionnaires. De peur de mettre en danger sa jeune épouse, il a préféré ne pas la mettre au courant de ses activités clandestines. Ils ont eu un bébé et le couple menait une vie heureuse. Alors que le bébé avait buit mois, des bommes armés ont fait irruption dans la maison en l'absence de Juan. Ils ont battu et violé Lucia et menacé de tuer l'enfant. Lucia a vécu cette agression avant tout comme une trahison de la part de Juan. Juan, submergé de culpabilité, ne pouvait plus faire appel à ses valeurs sociales et politiques pour comprendre et accepter ce qui s'était passé puisque c'est justement cette idéologie que Lucia rejetait maintenant en bloc. Il avait toujours su qu'il risquait d'être arrêté, torturé et peut-être tué mais était persuadé que sa foi dans une cause juste le soutiendrait. Il n'avait pas voulu imaginer qu'il pourrait mettre en danger ceux qu'il aimait le plus. Le couple s'est présenté à la clinique de Pointe-St-Charles avant de se séparer : ils ne pouvaient plus se parler. Peu à peu, de silence en silence, un lieu d'apaisement est apparu : ils ont commencé à fréquenter une église. Ils étaient tous deux catholiques mais n'y avaient jamais accordé beaucoup d'importance. Alors que les significations politiques et sociales étaient synonymes de trahison, les significations religieuses ont permis au couple de se retrouver autour de racines communes et de se rapprocher autour de la possibilité de rêver le futur de leur enfant.

Le travail de construction du sens se fait d'abord et avant tout dans les familles et au sein du groupe social auquel le réfugié s'identifie. Parfois cependant, comme dans le cas de Juan et Lucia, les mécanismes traditionnels, politiques et sociaux n'arrivent plus à jouer leur rôle et une intervention extérieure devient nécessaire.

\section{Intervenir : du domaine politique à l'espace thérapeutique}

Les lignes directrices de l'intervention proposée varient selon que l'on favorise une approche centrée sur la maladie au niveau des individus ou sur la pathologie au niveau social, sur une vision phénoménologique des symptômes ou sur une compréhension du sens des symptômes et des forces associées à l'histoire du réfugié et de sa communauté.

Le courant dominant, dans les pays occidentaux (Amérique du Nord, Danemark, pays nordiques), favorise une structuration classique de 
services psychiatriques, sociaux et scolaires adaptés aux problèmes spécifiques des réfugiés en tant qu'individus. Il s'agit, dans cette optique, d'insuffler une sensibilité culturelle aux outils thérapeutiques occidentaux comme la pharmacothérapie, le counseling et l'éducation (Westermeyer, 1991). Les professionnels intervenant auprès des réfugiés doivent acquérir une formation à l'intervention transculturelle et une expérience au sujet du trauma et de la réponse post-traumatique. Ce modèle médical est centré autour de la notion du SSPT et les approches cognitives dominent (March et al., 1998). Il s'agit essentiellement de comprendre que les symptômes ressentis constituent une réponse normale à l'expérience vécue, de reprendre le contrôle sur son vécu et de rétablir des liens de confiance à partir d'une appréhension du réel permettant de contrer l'impression que le traumatisme va se répéter (Pynoos et Eth, 1985). L'intervention individuelle, centrée autour d'une verbalisation de l'expérience dans un contexte thérapeutique, est donc la pierre d'angle du traitement des victimes de violence et de stress extrême. Richman (1993) questionne l'universalisation d'une telle approche auprès des personnes qui ont subi des situations de guerre, mettant de l'avant que le déni et le non-dit peuvent parfois être des stratégies utiles pour faire face à une situation de stress prolongé. Elle mentionne aussi que la relation thérapeutique duelle n'est pas nécessairement acceptable ou appropriée pour de telles situations, surtout si elle ne tient pas compte des dimensions collectives de l'expérience vécue. Le débat non résolu autour de la place du silence et du dévoilement, de la possibilité de travailler sur ou autour du trauma, pose la question du rythme de l'intervention et de la nécessité de savoir ne pas intervenir.

Le modèle clinique traditionnel est aussi interpellé par les dimensions politiques qui structurent non seulement le passé, mais le présent des communautés réfugiées. Face à une opinion publique ambivalente sinon hostile (Beiser, 1999 ; Moreau et al., 1999) et à des politiques migratoires qui visent souvent plus à dépister les fraudeurs qu'à protéger les réfugiés, les cliniciens ne peuvent pas se cantonner dans une position de « neutralité » sans devenir part de cette réalité qui agresse le réfugié. Un parti pris franc et résolu en faveur du droit d'asile et un engagement sous diverses formes, entre autres au niveau d'interactions avec les instances de l'immigration, est un préalable nécessaire à l'établissement d'une alliance thérapeutique. Cet engagement signe notre reconnaissance de la responsabilité collective que portent les thérapeutes, en tant que 
membres de la société hôte, et fait une place à la réalité sociopolitique dans l'espace thérapeutique.

\section{Bricoler : une solution}

La situation d'entre-deux dans laquelle se trouvent les familles réfugiées coupe les personnes des recours auxquels elles auraient pu avoir accès dans leur pays d'origine sans pour autant leur faciliter l'accès aux services du pays hôte. Ces services, s'ils sont accessibles linguistiquement et géographiquement (ce qui est loin d'être toujours le cas), le sont plus rarement sur le plan de l'ouverture à la différence, de la capacité des intervenants de se décentrer.

La contrepartie positive de ce constat, surtout vécu comme négatif, c'est que tout devient possible : l'absence de structures d'aide bien établies, même si elle peut être pénible, ouvre aussi la porte à la création de solutions nouvelles qui empruntent aux différents univers en présence. Or, aucun des modèles d'intervention existant jusqu'à présent ne propose une réponse complètement satisfaisante à la question de la violence organisée même si tous ont des approches potentiellement utiles. Ainsi le modèle médical, malgré les risques que comporte sa pathologisation de la souffrance sociale, propose des solutions psychothérapeutiques et psychopharmacologiques qui peuvent s'avérer une source de soulagement important (Keane et al., 1992).

Prédominant dans certains milieux en Amérique latine, le modèle politique met de l'avant que la force mobilisée par la souffrance due au traumatisme peut s'exprimer et devenir signifiante dans l'action politique : le témoignage devient un processus thérapeutique (Lira et Weinstein, 1984), puisqu'il devient un instrument de lutte et de protestation contre l'agresseur, en même temps qu'il est un geste de reconnaissance de la blessure et constitue, par là même, une rupture du silence imposée par la répression. L'intervention favorisée par ce courant se situe au carrefour d'une compréhension sociale et politique et d'une saisie psychodynamique et se fonde sur une articulation entre l'espace thérapeutique et l'espace sociopolitique (Vinar et Vinar, 1989). Toutes les communautés ne se reconnaissent cependant pas dans une interprétation politique de leurs expériences.

De plus en plus, quoique de façon encore marginale, on commence à reconnaître la valeur des modèles traditionnels dans les réactions face 
aux traumatismes. Ces modèles mettent de l'avant des savoirs traditionnels qui permettent de faire face à des situations de transgression et de « frayeur » comme le sont la violence extrême et les deuils difficiles. Que ce soit pour faire face à des morts non ritualisables (Eisenbruch, 1988 ; Campbell, 1992 ; Rechtman, 1992), pour effacer le poids du sang versé ou pour atténuer la douleur psychique ou physique, les savoirs traditionnels occupent une place incontournable à cause, entre autres, de leur impact sur la reconstruction du sens et du lien social. Tout en reconnaissant leur potentiel, il faut se garder d'idéaliser les ressources traditionnelles qui n'ont souvent pas été conçues pour répondre à des phénomènes de violence massive, ou le modèle politique qui s'adresse à une situation où les agresseurs et les victimes peuvent être assez clairement identifiés mais qui devient beaucoup plus difficile à appliquer lorsque, les conflits s'éternisant, il y a internalisation de la violence jusqu'au non-sens.

La possibilité pour les familles réfugiées d'utiliser simultanément plusieurs recours complémentaires ou même opposés leur permet de reprendre un pouvoir sur la situation, et de réintroduire un mouvement qui permet de sortir du temps suspendu, du temps immobile qui suit le traumatisme. Les équipes cliniques peuvent ici jouer un rôle de médiation, en facilitant la co-construction de solutions métisses qui reflètent la multiplicité des univers dans lesquels naviguent les réfugiés (Rousseau, 1998).

\section{Conclusion}

Au moment d'élaborer des programmes pour les réfugiés, le rôle des stratégies de reconstruction du sens et du lien social mises en place de l'intérieur par les réfugiés est trop fréquemment méconnu par les instances nationales et internationales qui les prennent en charge (Zarowsky, 1997). Cette méconnaissance de l'interaction dynamique entre les forces personnelles et collectives présidant à la reconstruction dans une société touchée par la violence peut conduire paradoxalement à nuire aux réfugiés, en mettant en place des structures d'aide qui entravent ou nuisent au développement de ces autres processus. Les interventions de la part des intervenants de la société hôte peuvent également avoir une place car il faut aussi se garder de trop idéaliser les ressources de sociétés dont le tissu social a été très atteint. Ces interventions devraient cependant se fonder, d'abord et avant tout, sur les savoirs dont sont porteuses ces communautés pour les potentialiser et les compléter. 


\section{Références}

Becker, D., Lira, E., Castillo, M. I., Gomez, E. et Kovalsky, J. 1990. «Therapy with victims of political repression in Chile: The challenge of social reparation », Journal of Social Issues 46 (3) : 133-149.

BeISER, M. 1999. Strangers at the gate-The "Boat People's" First Ten Years in Canada, Toronto, Buffalo, London : University of Toronto Press.

Beiser, M., Dion, R. et Gotowiec, A. 1998. «Depression and Conduct Disorder in Native and Non-Native Children ", Journal of the American Academy of Child and Adolescent Psychiatry 37 (7) : 736-742.

CAmpbell, H. L. 1992. «Southeast Asian Refugee Psychotherapy : Progression of Dreams. A Clinical Study », SPC, Narini, Italie.

De Certeau, M. 1987. «Corps torturés, paroles capturées », Cabiers pour un temps.

EISENBRUCH, M. 1988. «The Mental Health of Refugee Children and their Cultural Development », International Migration Review 22 (2) : 282-300.

Keane, T. M., Albano, A. M. et Blake, D. D. 1992. "Current Trends in the Treatment of Post-Traumatic Stress Symptoms », p. 363-401 in Torture and its consequences: current treatment approaches, sous la direction de M. Basoglu. Cambridge : Cambridge University Press.

KIRMAYER, L. J. 1996. "Landscapes of Memory Trauma, Narrative, and Dissociation », p. 173-198 in Tense Past Cultural Essays in Trauma and Memory, sous la direction de P. A. M. Lambek. New York : Routledge.

Kleinman, A. et Kleinman, J. 1997. « The Appeal of Experience; The Dismay of Images: Cultural Appropriations of Suffering in Our Times », p. 1-24 in Social Suffering, sous la direction de V. D. Arthur Kleinman et Margaret Lock. Berkeley : University of California.

Kordon, D., Edelman, L., Lagos, D., Nicoletti, E., Kersner, D. et Groshaus, M. 1992. «Torture in Argentina », p. 95-107 in Torture and its Consequences: Current Treatment Approaches, sous la direction de M. Basoglu. Cambridge : Cambridge University Press.

LiRA, E. et Weinstein, E. 1984. Psicoterapía y represión politica, Mexico : Siglo Veintiu.

Macksoud, M. S., Dyregrov, A. et Raundalen, M. 1993. «Traumatic War Experiences and Their Effects on Children », p. 625-633 in International Handbook of Traumatic Stress Syndromes, sous la direction de J. P. Wilson et B. Raphael. New York : Plenum Press.

March, J. S., Amaya-Jackson, L., Murray, M. C. et Schulte, A. 1998. «Cognitive-Behavioral Psychotherapy for Children and Adolescents With Posttraumatic Stress Disorder After a Single-Incident Stressor », Journal of American Child and Adolescent Psychiatry 37 (6) : 585-593.

MAROTTE, C. 1995. « The Phenomenon of Torture : Effect and Consequence », p. 232 in Our Midst. Educational Aids to Work with Survivals of Torture and Organized Violence. A Reflective Interactive Approach, sous la direction de B. Misgeld. Toronto : Canadian Centre for Victims of Torture.

MARotTE, C. 1999. «Faut-il être une victime pour devenir un citoyen? », Prisme 28 : 8-16. 
Mekki-Berrada, A., Rousseau, C. et Bertot, J. (soumis). "Research on Refugees : Means of Transmitting Suffering and Forging Social Bonds », International Journal of Mental Health.

Moreau, S., Rousseau, C. et MeKKI-Berrada, A. 1999. «Politiques d'immigration et santé mentale des réfugiés : profil et impact des séparations familiales », Nouvelles pratiques sociales 11 et 12 (1 et 2) : 177-196.

Nathan, T. 1988. «La migration des âmes », Nouvelle Revue d'Ethnopsychiatrie $11: 25-42$.

PRIMO-LÉVI 1995. Le Devoir de mémoire, Paris: Mille et une nuits (La petite collection).

PYNOOS, R. S. et ETH, S. 1985. « Children traumatized by witnessing acts of personal violence : Homicide, rape or suicide behavior », p. 168-186 in Posttraumatic stress disorder in children, sous la direction de R. S. Pynoos et S. Eth. Washington, DC : American Psychiatric Press.

PYNOOS, R. S. et NADER, K. 1993. "Issues in the Treatment of Posttraumatic Stress in Children and Adolescents », p. 535-549 in International Handbook of Traumatic Stress Syndromes, sous la direction de J. P. Wilson et B. Raphael. New York : Plenum Press.

ReCHTMAN, R. 1992. "L'apparition des ancêtres et des défunts dans les expériences traumatiques : introduction à une ethnographie clinique chez les réfugiés cambodgiens de Paris ", Cabiers d'Anthropologie et Biométrie Humaine $10(1-2): 1-19$.

RICHMAN, N. 1993. « Annotation: Children in situations of political violence », Journal of Child Psychology and Psychiatry 34 (8) : 1286-1302.

RousSEAU, C. 1998. «À propos de la violence organisée - Analyses et comptes rendus », Cabiers d'Études africaines 38 (2-4) : 674-676.

Rousseau, C., Drapeau A. et CORIN, E. 1992. « Premigratory and migratory risk factors for refugee children », Communication présentée d̀ l'Académie canadienne de pédopsychiatrie, Montréal.

Rousseau, C., Morales, M. et Foxen, P. (sous presse). « Going home: Giving voice to memory strategies of young Mayan refugees who returned to Guatemala as a community », Culture, Medicine and Psychiatry.

SKIDMORE, M. 1994. "The Politics of Space and Form: Cultural Idioms of Resistance and Re-membering in Cambodia », Santé, Culture, Health 10 (1 et 2) : 35-59.

SummerfielD, D. 1998. «The Social Experience of War and Some Issues for the Humanitarian Field », p. 9-37 in Retbinking the Trauma of War, sous la direction de P. J. Braeken et C. Petty. London, New York : Free Association Books Ltd.

TAUSSIG, M. 1992. «Violence and Resistance in the Americas: The Legacy of Conquest », p. 37-53 in The Nervous System. Grande-Bretagne : Routledge, Chapman and Hall.

TERR, L. C. 1991. «Chilhood traumas: An outline and overview », American Journal of Psychiatry 148 : 10-21.

VINAR, M. et VINAR, M. 1989. Exil et torture, Paris : Éditions Denoël.

WeSTERMEYER, J. 1991. "Psychiatric services for refugee children: An overview », p. 127-162 in Refugee children: Theory, research and services, sous la 
direction de F. L. Ahearn Jr. et J. L. Athey. Baltimore: Johns Hopkins University Press.

Yehuda, R. et McFarlane, A. C. 1995. "Conflict Between Current Knowledge About Posttraumatic Stress Disorder and Its Original Conceptual Basis », The American Journal of Psychiatry 152 (12) : 1705-1713. YounG, A. 1995. The Harmony of Illusions. Inventing Post-Traumatic Stress Disorder, Princeton, N.J.: Princeton University Press.

ZarowSKY, C. 1997. "Trauma, Development, Dispossession: "Telling the Story" of Refugees and Suffering in Somali Ethiopia », Refuge 16 (5) : 11-17. 